\title{
Satisfacción de pacientes con cicatrices atróficas de acné tratados con láser ablativo y/o técnicas quirúrgicas.
}

Hilda Rojas $\mathrm{P}^{1}$, Nadia Vega $\mathrm{A}^{2}$, Nelson Lobos $\mathrm{G}^{2}$, Daniela Carvajal $\mathrm{A}^{3}$.

'Servicio de Dermatología, Hospital Clínico Universidad de Chile, Santiago, Chile; ²Departamento de Dermatología, Facultad de Medicina, Universidad de Chile, Santiago, Chile; ${ }^{3}$ Centro de Salud Familiar Apoquindo, Santiago, Chile.

\section{Resumen}

Las cicatrices atróficas constituyen una complicación frecuente del acné vulgar. Existen múltiples tratamientos quirúrgicos, aunque ninguno ha demostrado ser satisfactorio. Se realizó un estudio transversal retrospectivo de 47 pacientes con cicatrices atróficas de acné intervenidos mediante Cirugía Punch (CP) y/o Láser CO2 $10.600 \mathrm{~nm}$ (LCO), a quienes se aplicó un cuestionario validado entre Abril y Septiembre de 2014. El 63,8\% recibió tratamiento solo con LCO, el $10,6 \%$ solo con CP y el $25,6 \%$ recibió ambos tratamientos. Respecto al grado de satisfacción, el $9 \%$ refirió un puntaje de 10 luego del procedimiento, el $32 \%$ entre 7-9, el $43 \%$ entre $4-6$ y un $17 \%$ entre $0-3$. El $66 \%$ de los pacientes refirió que los efectos se mantienen en el tiempo y el $21 \%$ reportó efectos adversos. El tratamiento quirúrgico de las cicatrices atróficas de acné con técnicas como CP y/o LCO consigue grados de satisfacción moderados, con efectos mantenidos a largo plazo y escasos efectos adversos.

Palabras claves: Cicatrices Atróficas; Cirugía Punch (CP); Láser CO2 $10.600 \mathrm{~nm}$.

\section{Summary}

Atrophic scars are a common complication of acne vulgaris. There are multiple surgical treatments, but none have proved satisfactory. A retrospective cross-sectional study of 47 patients with atrophic acne scars surgery was performed by Punch Surgery (CP) and / or CO2 Laser 10,600 nm (LCO), a validated questionnaire between April and September 2014 was applied. $63.8 \%$ received only LCO treatment, only $10.6 \% \mathrm{CP}$ and $25.6 \%$ received both treatments. Regarding the degree of satisfaction, $9 \%$ reported a score of 10 after the procedure, 32\% between $7-9,43 \%$ between $4-6$ and $17 \%$ 0-3. $66 \%$ of patients reported that the effects are maintained over time, and $21 \%$ reported adverse effects. Surgical treatment of atrophic acne scars with techniques such as CP and / or LCO achieved moderate degree of satisfaction with maintained long-term effects and few adverse effects.

Key words: Atrophic scars; Punch (CP) Surgery; Laser $\mathrm{CO} 2$ $10,600 \mathrm{~nm}$.

\section{Introducción}

El acné cicatricial $(A C)$ corresponde a una complicación frecuente del acné vulgar ${ }^{1}$, que resulta del daño inflamatorio de la unidad pilosebácea asociado a un inadecuado proceso de cicatrización. Su relevancia radica en que puede conducir a una desfiguración física importante, generando un impacto psicosocial profundo en los pacientes ${ }^{2}$.

Las cicatrices atróficas son el tipo más frecuente y se producen por la destrucción del colágeno secundaria a una respuesta inflamatoria persistente. Según su morfología, se clasifican en cicatrices tipo icepick (picahielo), rolling (circulares) y boxcar (rectangulares o en sacabocado) ${ }^{3}$.
El adecuado tratamiento del acné en etapas iniciales es la medida más importante para prevenir las secuelas cicatriciales ${ }^{4}$, ya que una vez formada la cicatriz, no es posible eliminarla por completo. Sin embargo, es frecuente que los pacientes consulten cuando las lesiones cicatriciales ya se encuentran establecidas, por lo que el conocimiento de las distintas opciones terapéuticas es fundamental para la práctica dermatológica.

La elección del tratamiento debe ser individualizada, considerando la severidad de las lesiones, las expectativas del paciente, el perfil de efectos secundarios, el equipamiento 
disponible y los costos del tratamiento ${ }^{5}$. En la literatura, se han descrito múltiples alternativas de manejo médico o quirúrgico que si bien permiten mejorar el aspecto de las cicatrices ${ }^{6}$, ninguna por sí sola ha demostrado ser completamente eficaz, segura y satisfactoria.

El uso de laser ablativo y técnicas quirúrgicas como escisión elíptica, escisión o elevación por punch y subcisión ${ }^{3}$, han demostrado en diversos estudios internacionales una mejoría clínica importante de las cicatrices atróficas de acné ${ }^{7,8}$. Sin embargo, en nuestro país es escasa la evidencia respecto al éxito del tratamiento en $\mathrm{AC}$, a pesar de que constituye una patología prevalente en la población general.

El presente estudio tiene como objetivo caracterizar a la población que se ha realizado procedimientos de cirugía punch (CP) y/o láser CO2 10.600 nm (LCO) en el servicio de dermatología del Hospital Clínico de la Universidad de Chile $(\mathrm{HCUCH})$ y evaluar el grado de satisfacción con el tratamiento recibido.

\section{Materiales y Método}

Se realizó un estudio transversal retrospectivo en pacientes con cicatrices atróficas de acné intervenidos mediante CP y/o LCO en el servicio de dermatología del HCUCH desde el año 2002 hasta 2014. Para evaluar el grado de satisfacción con los tratamientos recibidos, se utilizó un cuestionario validado, el cual fue aplicado entre Abril y Septiembre de 2014.

Las variables analizadas fueron edad, sexo, técnica utilizada, puntaje de satisfacción de acuerdo a una escala de 1 a 10 (a mayor puntaje, mayor satisfacción), mantención del efecto terapéutico, efectos adversos y recomendación del procedimiento a otros pacientes.

Este trabajo contó con el consentimiento informado de los pacientes encuestados, resguardándose la confidencialidad y el anonimato de los datos obtenidos, siendo éstos utilizados sólo para los objetivos del presente estudio.

En relación al análisis estadístico, para las variables continuas se determinó la normalidad mediante la prueba de ShapiroWilk, en caso de presentar una distribución normal se representó mediante media y desviación estándar, y en caso de distribución no normal, mediante mediana y rango intercuartílico. Las variables cualitativas se representaron mediante frecuencia absoluta y relativa. La comparación del puntaje de satisfacción entre las tres alternativas terapéuticas (LCO, CP 0 ambas) se realizó mediante es test de ANOVA y la comparación de la satisfacción entre el grupo que transcurrió antes o después de un año entre el procedimiento y el cuestionario, se realizó mediante la prueba de T de Student de muestras independientes. Se utilizó un nivel de significancia de un 0,05 y se utilizó el programa STATA $13 \otimes$ (StataCorpLP, Texas, USA).

\section{Resultados}

De un total de 47 pacientes encuestados, el $51 \%$ fueron hombres y el $49 \%$ mujeres, con mediana de edad de 29 años (24-33 años).

En relación a los procedimientos, el $63,8 \%$ de los pacientes recibió tratamiento solo con $\mathrm{LCO}$, el $10,6 \%$ solo con CP y el $25,6 \%$ recibieron ambos tratamientos. El $74 \%$ de los pacientes fue intervenido hace más de un año al momento de la encuesta.

Respecto al grado de satisfacción de los pacientes, el $9 \%$ refirió un puntaje de 10 luego del procedimiento, el 32\% entre 7-9, el $43 \%$ entre $4-6$ y un $17 \%$ entre $0-3$ (Tabla 1 ).

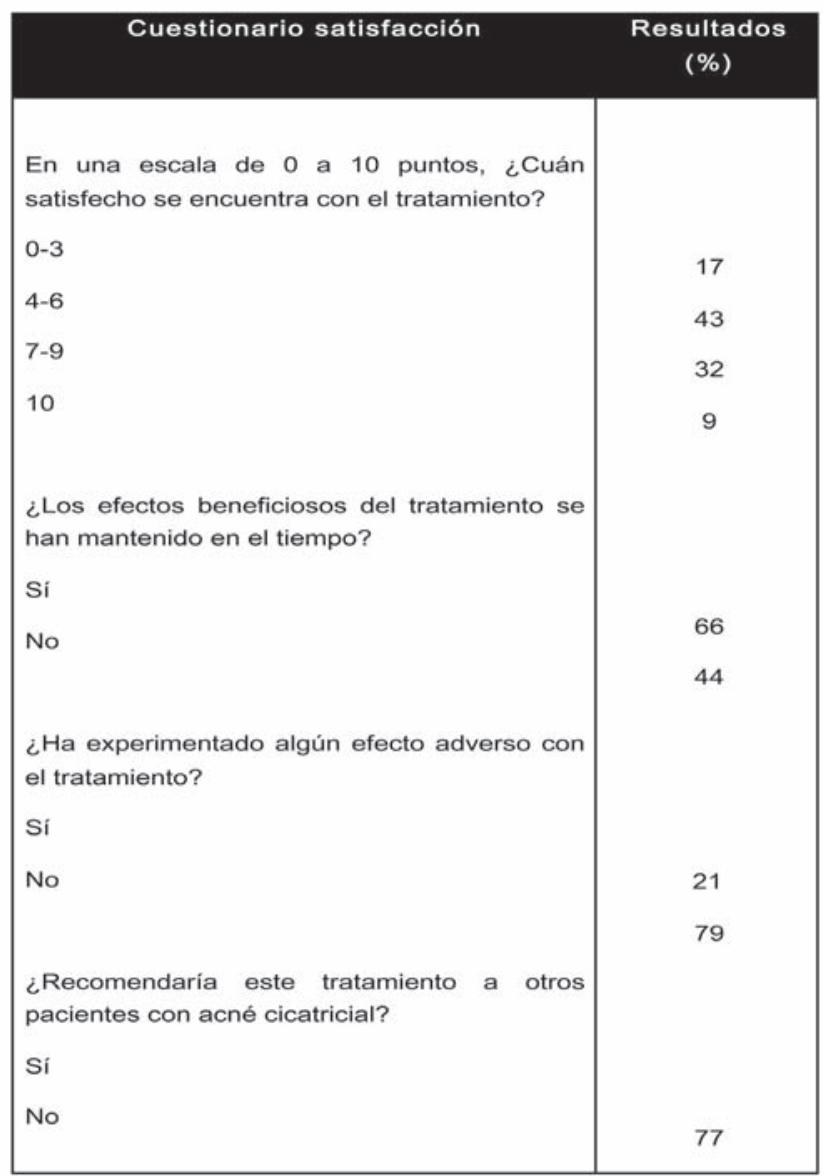

Tabla 1. Resultados del cuestionario de satisfacción. 


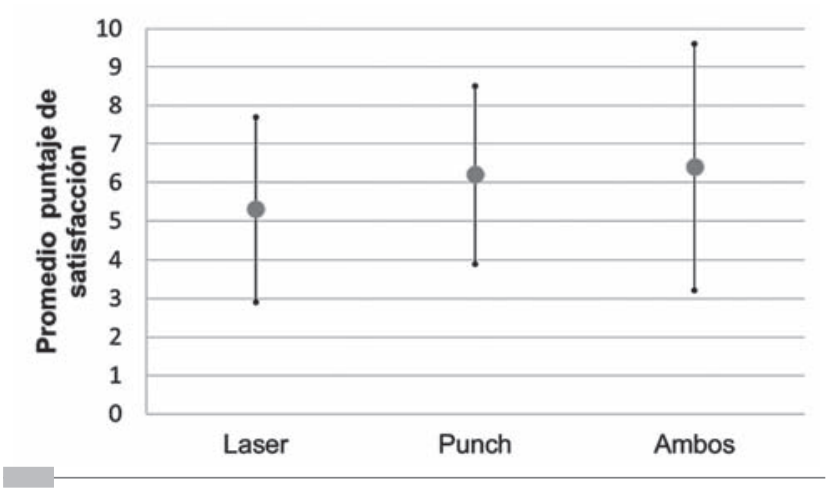

Figura 1. Promedio y desviación estándar del puntaje de satisfacción según terapia.

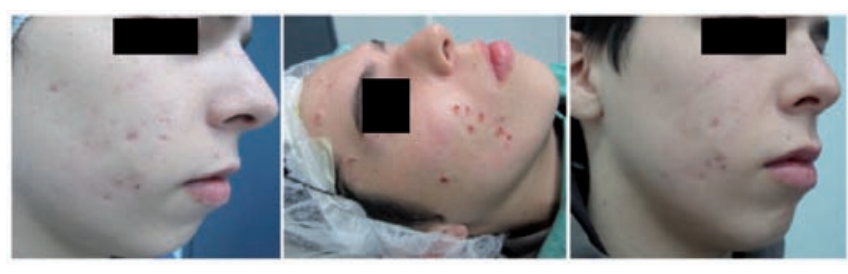

Figura 2. (a) Previo a CP, (b) post operatorio inmediato, (c) 3 meses después.

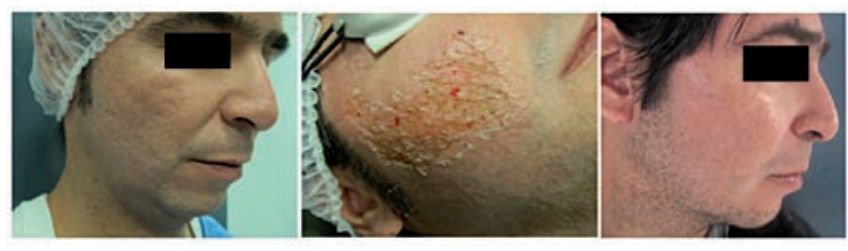

Figura 3. (a) Previo a sesión de LCO, (b) post operatorio inmediato, (c) 45 días después de la sesión.

Al analizarlo por técnica empleada, se observa que el puntaje promedio de satisfacción en los que recibieron solo terapia láser fue $5,3( \pm 2,4)$, solo cirugía punch fue $6,2( \pm 2,3)$ y ambas técnicas fue $6,4( \pm 3,2)$, sin diferencias significativas entre los grupos ( $p=0,47$ ) (Figuras 1,2 y 3). El puntaje promedio de satisfacción de los pacientes intervenidos hace más de un año fue $5,5( \pm 2,4)$ y en los que se realizó hace menos de un año fue $6( \pm 2,9)$, sin diferencias significativas entre ambos grupos $(p=0,57)$.

El $66 \%$ de los pacientes refirió que los efectos del tratamiento se mantienen en el tiempo y el $77 \%$ lo recomendaría a otros pacientes. En relación a los efectos adversos, fue, reportado en el $21 \%$ de los encuestados, siendo los más frecuentes el dolor y el eritema prolongado (Tabla 1).
Al analizar el grupo de pacientes que reportaron un puntaje de satisfacción mayor o igual a 7, se evidenció una asociación significativa con efectos mantenidos $(p<0,01)$, ausencia de efectos adversos $(p<0,01)$ y recomendación a otros pacientes $(p=0,03)$.

\section{Discusión}

Las secuelas del AC son frecuentes, existiendo en la actualidad múltiples alternativas de tratamiento. En nuestro estudio, la mayoría de los pacientes reportó un grado de satisfacción moderado frente al uso de LCO, CP o ambas terapias, lo cual se correlaciona con lo descrito en la literatura. Una revisión sistemática sobre el uso de LCO publicada por Jordan et al. reportó un promedio de mejoría que iba desde el $25 \%$ al $81 \%^{7}$. En 1999, Walia et al. realizaron un estudio prospectivo de LCO en 60 pacientes, observándose un $69 \%$ de mejoría promedio al mes de tratamiento, la cual continuó por al menos 18 meses alcanzando un $75 \%$ de mejoría ${ }^{8}$. Por lo tanto, el LCO es un procedimiento efectivo, que mejora significativamente el aspecto de las cicatrices atróficas tanto a corto como a largo plazo.

Respecto a la evidencia del tratamiento combinado, Grevelink et al., realizó un estudio de 21 pacientes donde se observó una importante mejoría cuando el láser fue aplicado luego de la escisión por punch. La ventaja de la técnica combinada se sustenta en que la CP elimina los componentes profundos de la cicatriz permitiendo el tratamiento superficial con láser en un menor número de sesiones ${ }^{9}$.

En relación a las reacciones adversas, el dolor y eritema prolongado fueron los reportados con mayor frecuencia en nuestro estudio. El trabajo publicado por Jordan et al. sobre el uso de LCO mostró una duración promedio del eritema de 6-16 semanas ${ }^{7}$, mientras que Whalia et al 8. reportaron una duración promedio de 14 semanas.

Aunque las alteraciones de la pigmentación (hiper o hipopigmentación) son una complicación frecuente del tratamiento con LCO, descritas hasta en el $44 \%$ de los pacientes ${ }^{7}$, no fueron reportadas en nuestro estudio. Esto podría explicarse por el fototipo de los pacientes intervenidos, dado que suelen presentarse con más frecuencia en fototipos oscuros ${ }^{10}$.

Respecto a otros efectos adversos reportados en la literatura como retraso en la cicatrización o cicatrices adicionales, dermatitis, milia, erupciones acneiformes, infección y telangiectasias, no fueron reportados en nuestro estudio.

Consideramos que uno de los principales determinantes en la aparición y tolerancia a los efectos adversos del LCO son la experiencia del operador y la adecuada selección del paciente. En nuestro estudio, todos los procedimientos fueron realizados por el 
mismo operador, quien cuenta con una amplia experiencia en la técnica y uso del dispositivo.

En relación a las limitaciones de nuestro estudio, las cuales se deben principalmente a su diseño retrospectivo, mencionamos la falta de estandarización del periodo de tiempo transcurrido entre el procedimiento y el día de la entrevista, lo cual podría alterar la percepción de los resultados, y la ausencia de descripción del tipo de cicatriz atrófica tratada, ya que como se ha observado en diversos estudios ${ }^{2}$, existen tratamientos específicos según el tipo de cicatriz, por lo cual nuestros resultados no podrían ser extrapolados a cada una de ellas.

Aunque el objetivo de este estudio fue evaluar el grado de satisfacción de los pacientes con acné cicatricial, independiente de la gravedad y/o número de lesiones, se observó que los pacientes con acné cicatricial más severo, tuvieron un mayor grado de satisfacción que los pacientes de menor severidad. Es posible que el nivel de expectativas fuera diferente en ambos grupos y explicara este fenómeno, sin embargo, son necesarios nuevos estudios que estandaricen la severidad del acné cicatricial antes y después de los procedimientos para confirmar esta hipótesis.

Del mismo modo, es importante considerar que nuestro estudio no refleja necesariamente la experiencia de otros centros donde se realizan este tipo de procedimientos quirúrgicos, ya que existen diferencias en cuanto al equipamiento y el grado de entrenamiento del personal, ambos factores fundamentales para la obtención de buenos resultados cosméticos.

\section{Conclusión}

El AC es una patología frecuente cuyo manejo constituye hoy en día un desafío terapéutico en la práctica dermatológica. En la actualidad, existen múltiples tratamientos disponibles tanto médicos como quirúrgicos, siendo necesario un enfoque multidisciplinariro y una adecuada selección del paciente para lograr los mejores resultados. Independiente de la técnica utilizada se debe comprender que sólo existe una mejoría de las cicatrices del acné, y nunca una curación total.

\section{Referencias bibliográficas}

1. Layton AM, Henderson CA, Cunliffe WJ. A clinical evaluation of acne scarring and its incidence. Clin Exp Dermatol 1994; 19:303.

2. Koo J. The psychosocial impact of acne: patients' perceptions. J Am Acad Dermatol 1995;32(Suppl):S26-30.

3. Jacob Cl, Dover JS, Kaminer MS. Acne scarring: a classification system and review of treatment options. J Am Acad Dermatol 2001; 45:109.

4. Goodman GJ. Treatment of acne scarring. Int J Dermatol. 2011 Oct;50(10):1179-94.

5. Rivera AE. Acne scarring: A review and current treatment modalities. J Am Acad Dermatol. 2008 Oct; 59(4):659-76.

6. Gozali MV, Zhou B. Effective treatments of atrophic acne scars. J Clin Aesthet Dermatol. 2015 May;8(5):33-40.

7. Jordan R, Cummins C, Burls A. Laser resurfacing of the skin for the improvement of facial acne scarring: a systematic review of the evidence. $\mathrm{Br} J$ Dermatol. 2000 Mar;142(3):413-23.

8. Walia S, Alster TS. Prolonged clinical and histological effects from $\mathrm{CO} 2$ laser resurfacing of atrophic acne scars. Dermatol Surg 1999;25:926-30.

9. Grevelink JM, White VR. Concurrent use of laser skin resurfacing and punch excision in the treatment of facial acne scarring. Dermatol Surg 1998;24:52730.

10. Jackson BA. Lasers in ethnic skin: a review. J Am Acad Dermatol. 2003 Jun; 48(6): 134-8. 